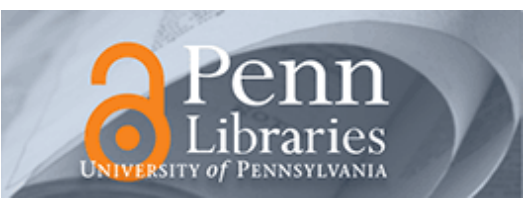

University of Pennsylvania

ScholarlyCommons

$9-18-2006$

\title{
Spectroscopic Studies of Fractal Aggregates of Silver Nanospheres Undergoing Local Restructuring
}

\author{
Sergei V. Karpov \\ Russian Academy of Sciences \\ Valeriy S. Gerasimov \\ Krasnoyarsk State Technical University \\ Ivan L. Isaev \\ Krasnoyarsk State Technical University \\ Vadim A. Markel \\ University of Pennsylvania, vmarkel@mail.med.upenn.edu
}

Follow this and additional works at: https://repository.upenn.edu/be_papers

Part of the Biomedical Engineering and Bioengineering Commons

\section{Recommended Citation}

Karpov, S. V., Gerasimov, V. S., Isaev, I. L., \& Markel, V. A. (2006). Spectroscopic Studies of Fractal Aggregates of Silver Nanospheres Undergoing Local Restructuring. Retrieved from https://repository.upenn.edu/be_papers/178

Suggested Citation:

Karpov, S.V., Gerasimov, V.S., Isaev, I.L. and Markel, V. (2006). Spectroscopic studies of fractal aggregates of silver nanospheres undergoing local restructuring. The Journal of Chemical Physics. 125, 111101.

(C) 2006 American Institute of Physics. This article may be downloaded for personal use only. Any other use requires prior permission of the author and the American Institute of Physics. The following article appeared in The Journal of Chemical Physics and may be found at http://dx.doi.org/10.1063/2229202.

This paper is posted at ScholarlyCommons. https://repository.upenn.edu/be_papers/178

For more information, please contact repository@pobox.upenn.edu. 


\title{
Spectroscopic Studies of Fractal Aggregates of Silver Nanospheres Undergoing Local Restructuring
}

\author{
Abstract \\ We present an experimental spectroscopic study of large random colloidal aggregates of silver \\ nanoparticles undergoing local restructuring. We argue that such well-known phenomena as strong \\ fluctuation of local electromagnetic fields, appearance of "hot spots" and enhancement of nonlinear \\ optical responses depend on the local structure on the scales of several nanosphere diameters, rather \\ than the large-scale fractal geometry of the sample. \\ Disciplines \\ Biomedical Engineering and Bioengineering | Engineering

\section{Comments} \\ Suggested Citation: \\ Karpov, S.V., Gerasimov, V.S., Isaev, I.L. and Markel, V. (2006). Spectroscopic studies of fractal aggregates \\ of silver nanospheres undergoing local restructuring. The Journal of Chemical Physics. 125, 111101. \\ (C) 2006 American Institute of Physics. This article may be downloaded for personal use only. Any other \\ use requires prior permission of the author and the American Institute of Physics. The following article \\ appeared in The Journal of Chemical Physics and may be found at http://dx.doi.org/10.1063/2229202.
}




\title{
Spectroscopic studies of fractal aggregates of silver nanospheres undergoing local restructuring
}

\author{
Sergei V. Karpov ${ }^{\text {a) }}$ \\ L.V. Kirensky Institute of Physics, Russian Academy of Sciences, Siberian Branch, Krasnoyarsk 660036, \\ Russia \\ Valeriy S. Gerasimov and Ivan L. Isaev \\ Department of Physics and Engineering, Krasnoyarsk State Technical University, Krasnoyarsk 660028, \\ Russia \\ Vadim A. Markel \\ Departments of Radiology and Bioengineering, University of Pennsylvania, Philadelphia, \\ Pennsylvania 19104
}

(Received 6 January 2006; accepted 22 June 2006; published online 18 September 2006)

\begin{abstract}
We present an experimental spectroscopic study of large random colloidal aggregates of silver nanoparticles undergoing local restructuring. We argue that such well-known phenomena as strong fluctuation of local electromagnetic fields, appearance of "hot spots" and enhancement of nonlinear optical responses depend on the local structure on the scales of several nanosphere diameters, rather than the large-scale fractal geometry of the sample. () 2006 American Institute of Physics.
\end{abstract} [DOI: $10.1063 / 1.2229202]$

Physical properties of surface plasmons (SPs) in disordered nanosystems have been the subject of intense studies in the past decade. ${ }^{1-4}$ The two objects that attracted most attention are disordered two-component composites, ${ }^{5-7}$ including two-dimensional percolation films, ${ }^{8}$ and aggregates of nanometer-sized noble metal spheres formed in colloidal solutions (colloidal aggregates). ${ }^{9}$ The latter are subject of this paper. Optical and, more generally, electromagnetic effects that were discovered in colloidal aggregates include giant enhancement of nonlinear-optical responses, ${ }^{9,10}$ inhomogeneous localization of electromagnetic eigenmodes, ${ }^{11,12}$ and optical memory. ${ }^{13,14}$

Perhaps, the most fundamental physical feature of electromagnetic interaction in colloid aggregates is inhomogeneous broadening. ${ }^{15}$ That is, different electromagnetic modes that can be excited in such aggregates (collective SP excitations) are resonant at different wavelengths which form a continuous band extending from the optical to the far-IR spectral region. ${ }^{16,17}$ In contrast, a single isolated silver nanosphere has a well defined narrow resonance centered at approximately $\lambda=400 \mathrm{~nm}$ (in hydrosols). ${ }^{1}$ The spectral shifts of the collective SP excitations are explained by the electromagnetic interaction of nanospheres. We view such excitations as collective even though not all nanospheres may effectively participate in a particular excitation mode. In fact, it was shown, that at any given electromagnetic frequency, there exist SP excitation which are delocalized over the whole sample, as well as excitations localized on a few neighboring nanospheres (hot spots). ${ }^{11,12}$ It was also shown, both experimentally and in simulations, that the locations of these hot spots are very sensitive to the electromagnetic fre-

\footnotetext{
${ }^{a)}$ Author to whom correspondence should be addressed. Electronic mail: karpov@iph.krasn.ru
}

quency and polarization of the incident wave. ${ }^{18}$

The spatial properties of the collective SP excitations in random colloidal aggregates have been studied in great detail (see Refs. 1-4 and references therein). However, little is known about the relation between the sample geometry and spatial properties of electromagnetic eigenmodes. Typically, the eigenmodes are obtained as solutions to the electromagnetic interaction problem. More specifically, an infinite matrix representing the electromagnetic interaction operator is truncated and diagonalized numerically. ${ }^{19}$ Each element of this matrix is completely defined by the sample geometry. In principle, the same is also true for the eigenmodes. Unfortunately, the mathematical dependence between elements of a large matrix and its eigenvectors can be very complex and, in the general case, not easily analyzable. Some approximate theories that directly relate the spatial characteristics and electromagnetic field inside the sample were based on the first Born ${ }^{20}$ and mean-field ${ }^{21}$ approximations, on few-body interaction approximation (binary ${ }^{15}$ or binary-ternary ${ }^{12}$ approximations), and various phenomenological scaling laws. ${ }^{15,22,23}$ The first Born and the mean-field approximations are not applicable to resonant excitation of collective SPs. The few-body approximations and the scaling laws proved to be very useful for qualitative theoretical description at the early stages of research, but increasingly more realistic simulations revealed that these approaches do not provide quantitative results.

We have recently argued that the locations of "hot spots" in random fractal aggregates are strongly correlated with the local anisotropy factor ${ }^{24}$ which quantifies the deviation of the local environment of a given nanosphere in an aggregate from the spherical symmetry. We have shown in simulations that sites with high local anisotropy are likely to coincide with the "hot spots." This concept allows one to make a 
qualitative prediction about location of the "hot spots" in a large aggregate without actually solving the electromagnetic problem. In this paper we present experimental evidence of this conjecture.

Direct measurement of the local anisotropy factor in three-dimensional samples is a difficult experimental task. However, it is possible to observe the influence of local restructuring on the electromagnetic properties of a large aggregate indirectly by studying IR absorption of large nanoaggregates as they undergo local restructuring. Enhancement of the IR absorption in an aggregate relative to that of an isolated nanosphere is an important effect explained by strong resonance interaction of different nanospheres. It can be directly linked to enhancement of local fields and the appearance of "hot spots". For example, the enhancement factor for surface-enhanced Raman scattering (SERS) by Raman-active molecules adsorbed on nanosphere surfaces is given by the ratio of the extinction cross section of an aggregate to that of an isolated nanosphere (see Ref. 2, Sect.7 and Ref. 3, Sect.3.6.2). This result is exact in the limit of large Raman shifts. If the Raman shifts are small enough so that the Raman signal is still within the aggregate absorption band, SERS is enhanced by an additional factor which is approximately equal to the ratio of the extinction cross section of an isolated nanosphere at its maximum and at the Raman wavelength. Additionally, a number of approximate formulas that relate enhancement factors for various nonlinear processes in aggregates and the enhancement of linear extinction are given in these two references. We note that evolution of Raman spectra of benzene molecules adsorbed on cold-deposited self-affine silver films has been studied directly $^{25}$ during the process of thermal annealing. It was observed that the Raman activity ceases long before the overall fractal structure of the sample collapses. This phenomenon is in agreement with what we report in this note and was explained by variation in the nanosphere size. We, however, argue here that the more pertinent change is that of the local anisotropy factor.

We have studied extinction spectra of silver fractal aggregates embedded in a polymer matrix under uniform contraction. Experimental samples were prepared as follows. First, silver hydrosol was prepared by reduction of $\mathrm{AgNO}_{3}$ by $\mathrm{NaBH}_{4}$ in water solution ${ }^{9}$ (electrolyte concentration 2.5 $\times 10^{-3} \mathrm{M}$ ). After this chemical reaction, the hydrosol is a colloidal solution of silver nanospheres $10-40 \mathrm{~nm}$ in diameter that undergo random Brownian motion and can stick on contact and form large fractal aggregates. The aggregation process was accelerated by irradiation of the hydrosol with the natural light for $4-10 \mathrm{~min} .{ }^{26}$ The overall size of aggregates was of the order of $1 \mu \mathrm{m}$ or larger as follows from TEM images. Next, the hydrosol containing aggregates of silver nanospheres was added into a water solution of gelatin. The volume fraction of silver in the resultant solution was $\sim 10^{-6}$. Next, the prepared solution was allowed to gelate. As a result, the metal nanoparticles became rigidly connected to the polymer matrix of the gel and could not move freely. We have prepared rectangular gelatin samples with initial dimensions of $1 \times 5 \times 10 \mathrm{~cm}^{3}$. At the next stage, the gel underwent gradual dehydration and the sample volume

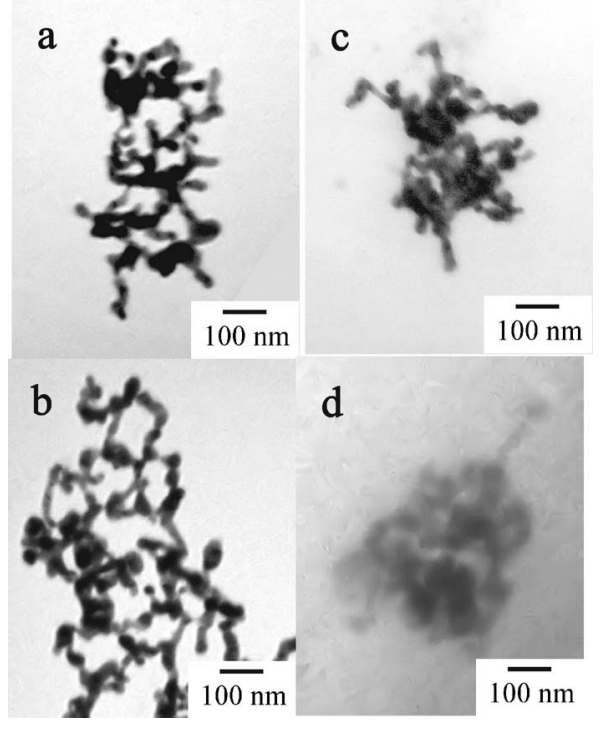

FIG. 1. Comparative electron micrographs of typical silver nanoaggregates before their embedding into the gelatin matrix $(a, b)$ and in slices of the gel in the final stage of the dehydration $(\mathrm{c}, \mathrm{d})$. The slice thickness is approximately 200-300 $\mathrm{nm}$ (c) and 500-700 $\mathrm{nm}$ (d). Low resolution in image (d) is explained by the relatively large slice thickness. Nanoaggregates shown in the left and right columns are not the same.

was reduced by the factor of $\approx 10$. This corresponds to linear contraction by the factor of $\approx 2.15$.

Electron micrographs of Ag nanoaggregates in the hydrosol and in thin slices of the gel (several hundreds $\mathrm{nm}$ thick) taken after the dehydration are shown in Fig. 1. It can be seen that, as the gelatin matrix undergoes contraction, the local geometrical structure of the aggregates changes. Neighboring nanospheres tend to form dense blobs which are spherically symmetric on average. Inside such blob, the local environment of a nanosphere is more similar to that in chaotic dense packing. Therefore, we expect that the local asymmetry factor is reduced in the restructured aggregates.

We have also simulated the restructuring process on a computer. Initially, a random two-dimensional (2D) aggregate with $N=700$ nanospheres and fractal dimension $D$ $\approx 1.46$ and a three-dimensional (3D) aggregate with $N$ $=2000$ nanospheres and fractal dimension $D \approx 1.83$ were generated using the method described in Ref. 19. Then the coordinates of each nanosphere were repeatedly multiplied by the factor 0.95 , while the sphere radii were kept constant. The contraction resulted in geometrical intersection of neighboring spheres. Then each sphere (in a predetermined order) was moved to the nearest possible position as to avoid its intersection with any other sphere in the aggregate. Next, the contraction by the factor 0.95 was repeated, and so on, until the overall linear contraction of the sample by the factor $\approx 2.5$ was achieved (approximately in 50 iterations). The stages of transformation of the aggregate are shown in Fig. 2. The local restructuring similar to the one seen in TEM images is clearly visible. But the global geometrical structure of aggregates remains approximately unchanged, within the limits imposed by the changing overall size of the aggregate. Note that the changes of local structure in 3D aggregates are partially obscured by the overlaps in $2 \mathrm{D}$ projection of a $3 \mathrm{D}$ object. This problem is not present in the case of $2 \mathrm{D}$ aggre- 


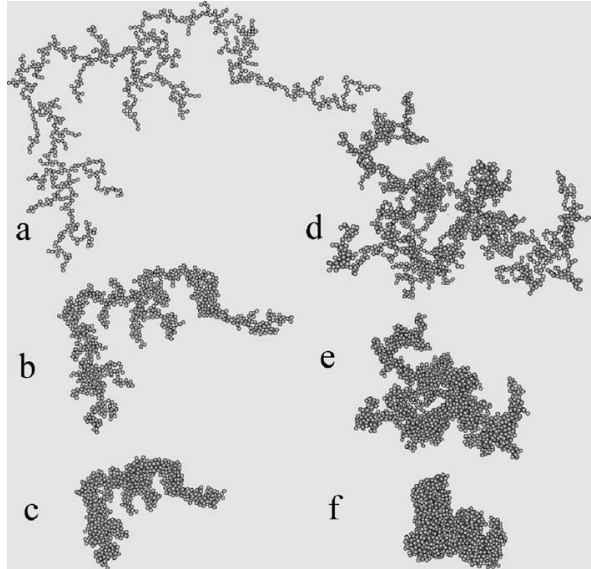

FIG. 2. Simulation of stages of transformation of 2D and 3D fractal aggregates embedded in a contracting gelatin matrix. Original 2D and $3 \mathrm{D}$ aggregates $(\mathrm{a}, \mathrm{d})$ after linear contraction by the factor $\sim 1.4(\mathrm{~b}, \mathrm{e})$ and $\sim 2.5(\mathrm{c}, \mathrm{f})$. For the 3D aggregate, the average value of the local anisotropy parameter $S$ defined in Ref. 24 are 1.03 (d), 0.94 (e) and 0.74 (f).

gates, which are shown in Fig. 2 for illustrative purpose.

The evolution of the absorption spectra of the samples as they undergo gradual contraction are shown in Fig. 3. Here the solid bold line (line 1) represents the absorption spectrum of silver hydrosol before aggregation, the solid thin line (line 2) the spectrum after aggregation and before embedding in the gelatin matrix. Dashed lines 3-5 describe different stages of dehydration of the matrix. These curves correspond to the absorption of silver aggregates; the absorption of pure gelatin was subtracted from the composite samples. It can be seen that, as the aggregates undergo restructuring due to the matrix contraction, the long wavelength spectral wing is sup-

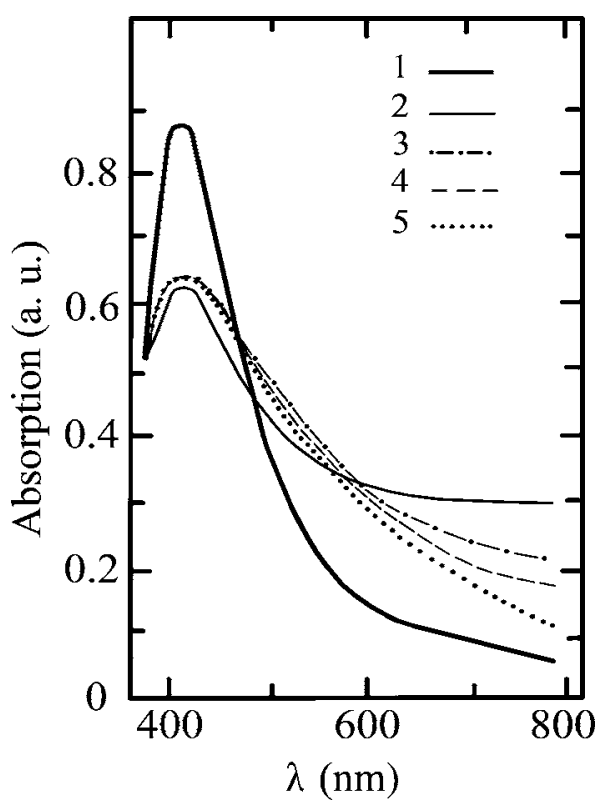

FIG. 3. Absorption spectra of silver hydrosol in non-aggregated stage (1) and in the final stage of aggregation (2), and of silver aggregates embedded in the gelatin matrix in different stages of dehydration of gelatin (3-5). Curves 3-5 show differential absorption spectra: the absorption of identical gelatin matrix without silver aggregates was subtracted. Absorption spectra are shown at the initial stage of freshly prepared matrix (curve 3), at the intermediate stage of dehydration (curve 4) and after full dehydration (curve 5). Curves 2-5 are normalized to common maximum. pressed. In particular, the absorption at $\lambda \approx 800 \mathrm{~nm}$ in the dehydrated gelatin matrix (curve 5 ) is reduced approximately by the factor of 2 compared to that in the original gelatin matrix (curve 3) and by the factor of 3 compared to aggregates in solution. We note that at that wavelength, the absorption in the dehydrated matrix is close to that of nonaggregated nanospheres in solution. This indicates that the effects related to inhomogeneous broadening, such as the giant local field fluctuations, appearance of "hot spots" and enhancement of nonlinear responses are strongly suppressed in the aggregates that underwent local restructuring. The spectral changes have been reproduced in a series of five independent experiments.

We note that similar spectral changes were observed during the process of self-organization (assembly of initially disordered aggregates in superlattices) of $\sim 5 \mathrm{~nm}$ sized silver particles in hexane solution ${ }^{27}$ and of $\sim 13 \mathrm{~nm}$ sized gold particles in water solution of mercaptoethyl alcohol (MEA). ${ }^{28}$ In the latter case, as the gold nanoparticles assembled in highlyanisotropic structures whose TEM images are shown in Figs. 2(c) and 2(d) of that reference, the IR spectral wing was enhanced. However, after the particles collapsed into a dense isotropic structure at high MEA concentration [Fig. 2(e) of Ref. 28], the IR spectral wing was suppressed. The effect can be naturally explained within the framework of the theory of Ref. 24. We note however, that the transition from highly anisotropic to more isotropic structures [e.g., from aggregates shown in Fig. 2(d) to those shown in Fig. 2(e) of Ref. 28] was also accompanied by a transition from a fractal to a non-fractal structure, similarly to the numerical experiment illustrated in Fig. 1 of Ref. 29, where the local field enhancement factor was compared for fractal and non-fractal aggregates. Thus, a definitive answer about the relative influence of large scale geometry (fractality) and the local anisotropy can not be deduced from the experiments of Refs. 27 and 28 and from numerical simulation of Ref. 29. Here we report experiments performed under more controlled conditions, i.e., when the local anisotropy factor is changed while the fractality is preserved. However, it should be admitted that the latter property (preservation of fractality) can be assessed only qualitatively from TEM images, or confirmed indirectly in numerical simulations. It is also important to keep in mind that the fractal aggregates forming under typical experimental conditions are usually characterized by highly anisotropic local structure. Thus, the theory of Ref. 24 does not contradict numerous previous observations of SERS enhancement in fractals, but rather provides a more detailed mechanism for such enhancement and, in addition, has predictive power. Namely, it allows one to identify sites with large local field enhancement in a large random aggregate without solving the electromagnetic interaction problem.

In summary, we have provided experimental evidence that the local anisotropy of the environment introduced in Ref. 24, rather than the large-scale geometrical structure, is one of the crucial factors for fluctuation and enhancement of local fields in random aggregates of nanospheres. There are, however, other important factors that contribute to this phenomenon. For example, the size of inter-particle gaps is expected to strongly influence both the linear spectra and the 
SERS. We did not control the size of interparticle gaps in the experiments described here. However, we have recently performed additional experiments in which this parameter was controlled and constant; the results do not contradict the findings reported here and will be published separately. Finally, the theoretical tools developed in Ref. 24 can be applied, for example, to detection of defects in photonic crystals, and in other areas of nanophotonics involving large disordered systems.

S.V.K., V.S.G., and I.L.I. acknowledge support from the following foundations of the Russian Federation: RFBR, Grant No. 05-03-32642, DSP, Grant No. 2.1.1.1814, SS, Grant No. 6612.2006.3, SB RAS, Grant No. 33. The authors are grateful to Dr. O.P. Podavalova for preparation of gelatin matrices and Dr. S.M. Zharkov for TEM images.

${ }^{1}$ U. Kreibig and M. Vollmer, Optical Properties of Metal Clusters (Springer, Berlin, 1995).

${ }^{2}$ M. I. Stockman, "Local fields localization and chaos and nonlinearoptical enhancement in clusters and composites," in Optics of Nanostructured Materials (Wiley, NY, 2000).

${ }^{3}$ V. M. Shalaev, Nonlinear Optics of Random Media: Fractal Composites and Metal-Dielectric Films (Springer, Berlin, 2000).

${ }^{4}$ V. I. Roldugin, Russ. Chem. Rev. 72, 913 (2003).

${ }^{5}$ D. J. Bergman, Phys. Rev. Lett. 44, 1285 (1980).

${ }^{6}$ D. E. Aspnes, Phys. Rev. Lett. 48, 1629 (1982).

${ }^{7}$ M. I. Stockman, K. B. Burlaev, and T. F. George, Phys. Rev. B 60, 17071 (1999).

${ }^{8}$ K. Seal, M. A. Nelson, Z. C. Ying, D. A. Genov, A. K. Sarychev, and V. M. Shalaev, Phys. Rev. B 67, 035318 (2003).

${ }^{9}$ A. V. Butenko, P. A. Chubakov, Y. E. Danilova, S. V. Karpov, A. K. Popov, S. G. Rautian, V. P. Safonov, V. V. Slabko, V. M. Shalaev, and M.
I. Stockman, Z. Phys. D: At., Mol. Clusters 17, 283 (1990).

${ }^{10}$ M. I. Stockman, V. M. Shalaev, M. Moskovits, R. Botet, and T. F. George, Phys. Rev. B 46, 2821 (1992).

${ }^{11}$ M. I. Stockman, L. N. Pandey, and T. F. George, Phys. Rev. B 53, 2183 (1996).

${ }^{12}$ M. I. Stockman, Phys. Rev. E 56, 6494 (1997).

${ }^{13}$ S. V. Karpov, A. K. Popov, S. G. Rautian, V. P. Safonov, V. V. Slabko, V. M. Shalaev, and M. I. Shtokman, JETP Lett. 48, 571 (1988).

${ }^{14}$ W. Kim, V. P. Safonov, V. M. Shalaev, and R. L. Armstrong, Phys. Rev. Lett. 82, 4811 (1999)

${ }^{15}$ V. A. Markel, L. S. Muratov, M. I. Stockman, and T. F. George, Phys. Rev. B 43, 8183 (1991).

${ }^{16}$ Y. E. Danilova, V. A. Markel, and V. P. Safonov, Atmos. Oceanic Opt. 6, 821 (1993).

${ }^{17}$ V. A. Markel, V. M. Shalaev, E. B. Stechel, W. Kim, and R. L. Armstrong, Phys. Rev. B 53, 2425 (1996).

${ }^{18}$ V. A. Markel, V. M. Shalaev, P. Zhang, W. Huynh, L. Tay, T. L. Haslett, and M. Moskovits, Phys. Rev. B 59, 10903 (1999).

${ }^{19}$ V. A. Markel, V. N. Pustovit, S. V. Karpov, A. V. Obuschenko, V. S. Gerasimov, and I. L. Isaev, Phys. Rev. B 70, 054202 (2004).

${ }^{20}$ J. E. Martin and A. J. Hurd, J. Appl. Crystallogr. 20, 61 (1987).

${ }^{21}$ M. V. Berry and I. C. Percival, Opt. Acta 33, 577 (1986).

${ }^{22}$ M. I. Stockman, L. N. Pandey, L. S. Muratov, and T. F. George, Phys. Rev. B 51, 185 (1995)

${ }^{23}$ V. M. Shalaev, M. I. Stockman, and R. Botet, Physica A 185, 181 (1992).

${ }^{24}$ S. V. Karpov, V. S. Gerasimov, I. L. Isaev, and V. A. Markel, Phys. Rev. B 72, 205425 (2005)

${ }^{25}$ C. Douketis, T. L. Haslett, Z. Wang, M. Moskovits, and S. Iannotta, J. Chem. Phys. 113, 11315 (2000).

${ }^{26}$ S. V. Karpov, V. V. Slabko, and G. A. Chiganova, Colloid J. 64, 425 (2002).

${ }^{27}$ A. Taleb, C. Petit, and M. P. Pileni, J. Phys. Chem. B 102, 2214 (1998).

${ }^{28}$ S. Lin, M. Li, E. Dujardin, C. Girard, and S. Mann, Adv. Mater. 17, 2553 (2005).

${ }^{29}$ V. M. Shalaev, E. Y. Poliakov, and V. A. Markel, Phys. Rev. B 53, 2437 (1996). 